D'autre part, la production de l'étang est aussi proportionnelle à la richesse nutritive du milieu, richesse que le pisciculleur améliore par l'apport de la chaux et l'usage d'engrais comme le ferait un herbager avant de charger en bétail sa prairie. Ios engrais sont les auxiliaires de l'éleveur de poisson comme des éleveurs de bêtes de boucherie (I).

\title{
LE SAUMON ET LE CONFLUENT DES GAVES DE PAU ET D'OLORON
}

\author{
Par M. SABATIER DE LACHADENĖDE \\ Conservateur des Eaux et Forîts
}

et M. DE DROUIN de BOUVILLE

Inspecteur des Eaux et Forets, en retraite

D'après nombre de témoignages dignes de foi, le Saumon en montée nuptiale qui entre dans l'Adour n'y effectue qu'un parcours limité, car, s'il ne l'a pas quitté à la jonction de la Nive, il le délaisse au confluent des Gaves réunis. On nomme ainsi le tronc commun des Gaves de Pau et d'Oloron, long de 4 kilomètres. Puis, les géniteurs en instance de fraye se répartissaient naguère entre ccs deux cours d'eau, mais assez inégalement. Le contingent du gave de Pau paraît avoir été toujours moindre.

Les choses se sont passées de la sorte jusqu'en igi7. $\Lambda$ partir de cette date, le Saumon est devenu de plus en plus rare dans le gave de Pau. Toutefois, en r 923 , on en pòchait encore 58 ct, en r924, 32, d'après les statistiques de pêche dressées par le Service local des Eaux et Forêts, statistiques forcément incomplètes, puisqu'elles ne tiennent compte que des déclarations bénévoles recueillies par les préposés forestiers.

Il paraît singulier, d'après ce qu'on sait de la biologie du Saumon, que l'abandon du Gave de Pau par ce migrateur ait été aussi rapide. On s'est demandé si des mesures ne pourraient être prises (construction d'échelles, travaux de repeuplement) pour rétablir la situation qui existait avant 1918.

L'Administration des Eaux et Forêts nous a confié, au printemps dernier, une mission pour l'étude de cette question, qui intéresse grandement la population paloise.

Nous avons parcouru le gave de Pau depuis Lourdes, le gave d'Oloron depuis cette dernière ville, jusqu'à leur confluent, et, au cours de cette tournée, rien ne fut observé qui permìt d'expliquer la préférence que le Saumon manifeste, depuis quelques années, pour le gave d'Oloron.

(1) Le Bulletin a donné des renseignements pratiques sur les " Distributeurs d'en-

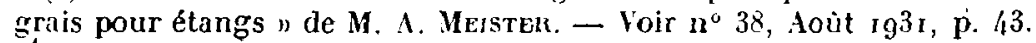

Article available at http://www.kmae=journal.org or http://dx.doi.org/10.1051/kmae:1933017 


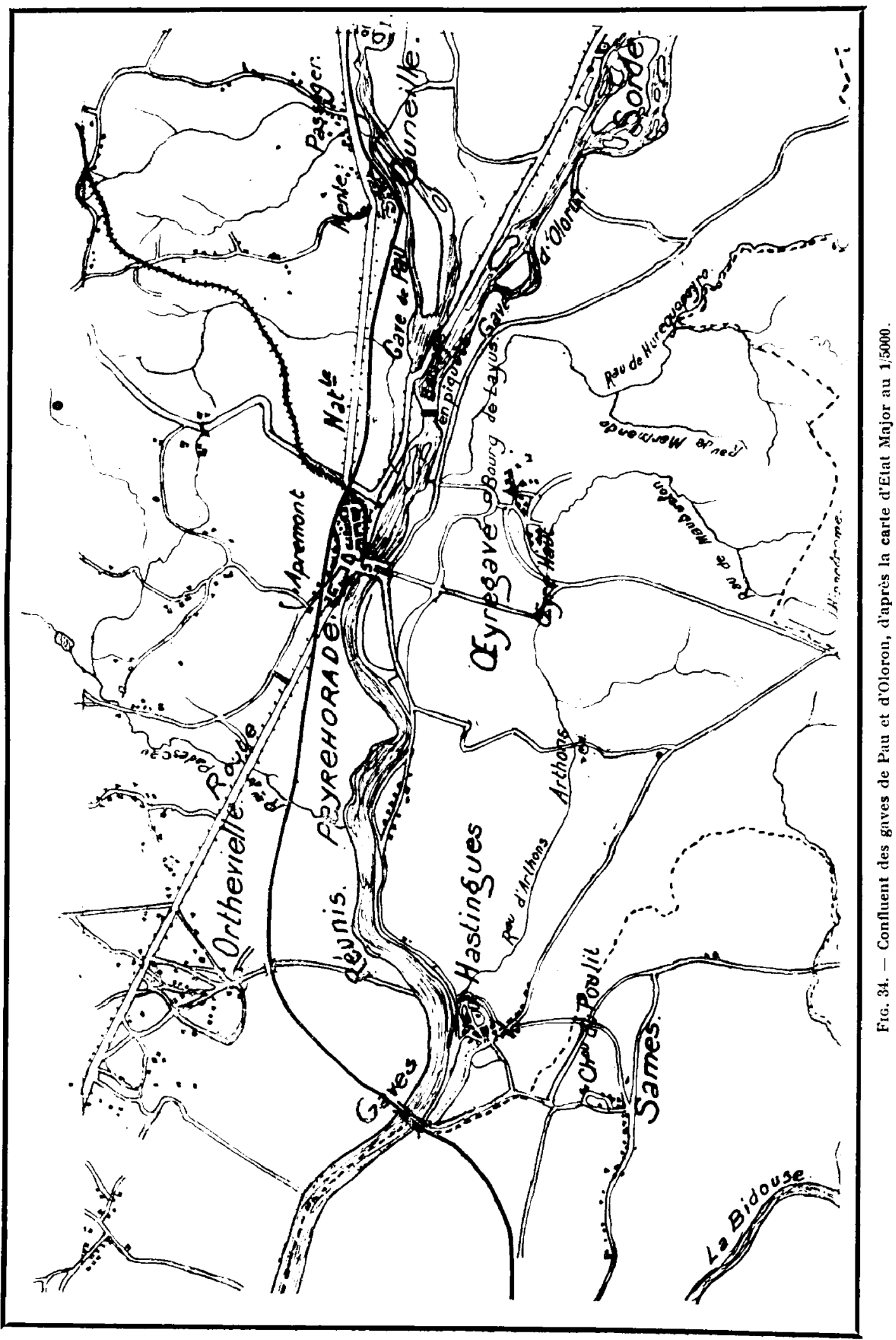


Toutefois, les personnes interrogées au cours de l'enquête furent d'avis que la construction d'un barrage infranchissable sur le gave de Pau, à Lastetarbe, un peu en dessous d'Orthez, et l'encombrement de ce mème gave à l'endroit de sa jonction avec celui d'Oloron, pourraient être les deux facteurs déterminants de la disparition du Saumon. A en croire certains, un banc de sable serait venu créer, au confluent, un premier obstacle ; puis, le barrage susdésigné, d'une hauteur de 6 à 7 mètres, mis en service en I 918 , aurait occasionné la stérilisation définitive du cours d'eau.

La première indication ne semblait pas mériter grande créance, surtout après examen des lieux. Quant à la suppression de toute remonte dans ie gave de lau, elle n'est pas absolument certaine. Il y a lieu d'observer qu'en aval de Ciastetarbe, cette rivière encaissée est très difficilement pèchable; peut-ètre, la fréquentation par le Saumon de cette partie du cours n'a-t-elle pas complètement cessé.

Néanmoins, pour ne rien négliger, nous convînmes de faire relever, par des sondages, le profil en travers de chacun des gaves au confluent, puis celui des gaves réunis à 20 mètres en aval.

Le travail, en raison de crues successives de l'Adour et de ses affluents, ne put être terminé que dans les premiers jours d'Août. Mais, aussitôt en possession des relevés de sondage, se révéla une anomalie. La coulière, du gave d'Oloron avait son fond à près d'un mètre en contre-bas de celle du gave de Pau.

Il apparaissait, par ailleurs, que le profil en long du dernier cours d'eau ne manifestait aucune tendance à se raccorder tangentiellement avec celui du premier.

Nous fìmes alors relever deux autres profils en travers, respectivement à 40 et 60 mètres en aval du confluent. Ils confirmèrent les indications des premiers. (Fig. 35).

Ces observations en provoquèrent d'autres, et il fut constaté, que la souille ou partic surcreusée prenait naissance dans le gave d'Oloron à 3 zoo mètres environ en amont du confluent ct que sa profondeur allait en croissant; elle atteint environ I mètre au confluent des deux gaves : 3 mètres au pont de Peyrehorade ; 4 mètres à roo mètres environ en aval de ce pont; puis 6 et ro mètres un peu en amont et en aval du pont de chemin de fer, au-dessous d'Hastingues. 'Toutefois, cette profondeur s'atténue ensuite à 5 mètres au bec des deux gaves, - confluent aves l'Adour, - pour se maintenir à ce chiffre dans ce dernier cours d'eau, jusqu'à sa jonction avec la Bidouze, à 3 kilomètres en aval de l'cmbouchure des gaves réunis. Les sondages n'ont pas été poussés plus loin (I).

La largeur de cette souille, peu considérable d'abord, va en grandissant; clle passe de $3^{\mathrm{m}} 50$ environ, au confluent des gaves de Pau et d'Oloron, à 50 mètres au point où les gaves réunis se jettent dans l'Adour.

(1) Ces mensurations peuvent subir une certaine variation, la marée moyenne occa sionnant des différences de niveau de $1 \mathrm{~m}$. 5o environ. 

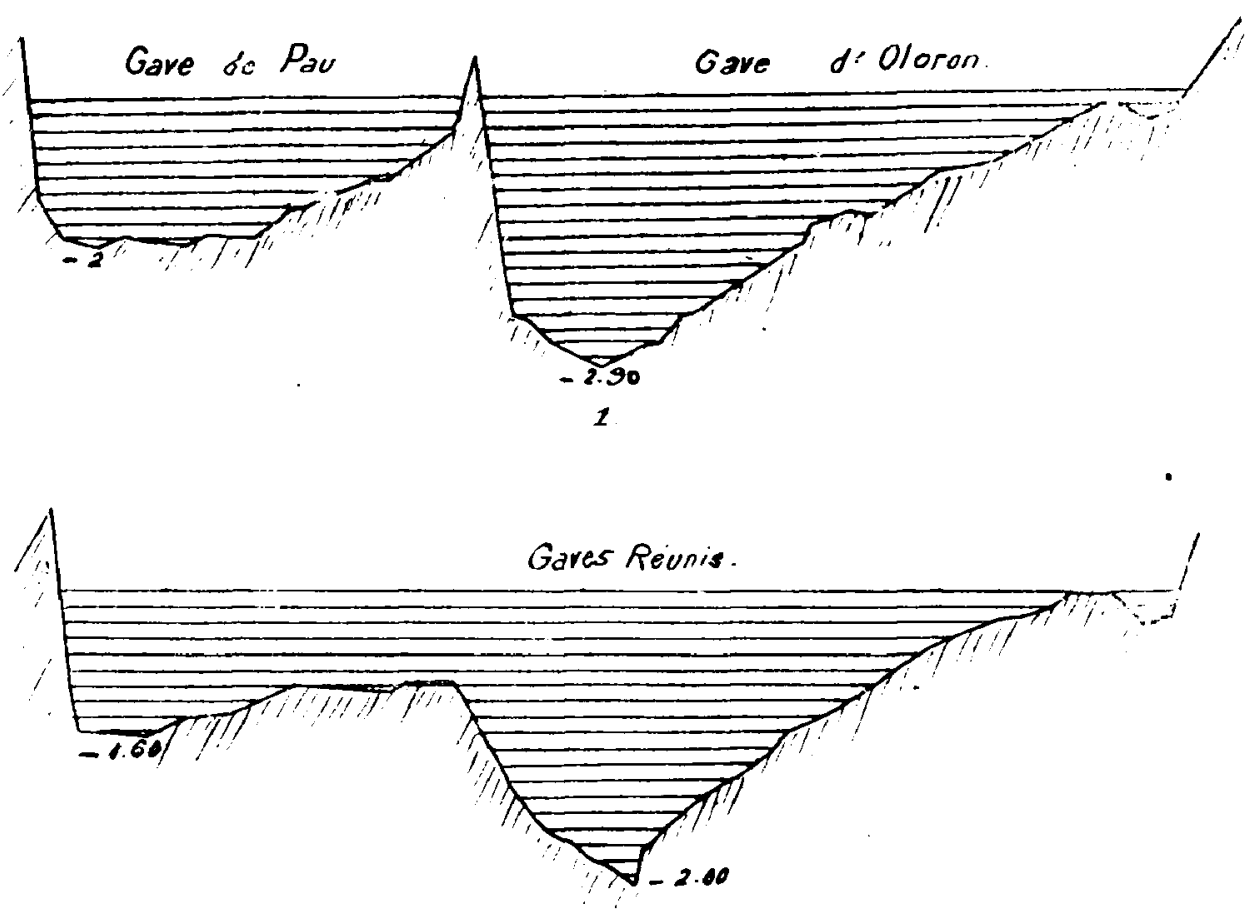

2
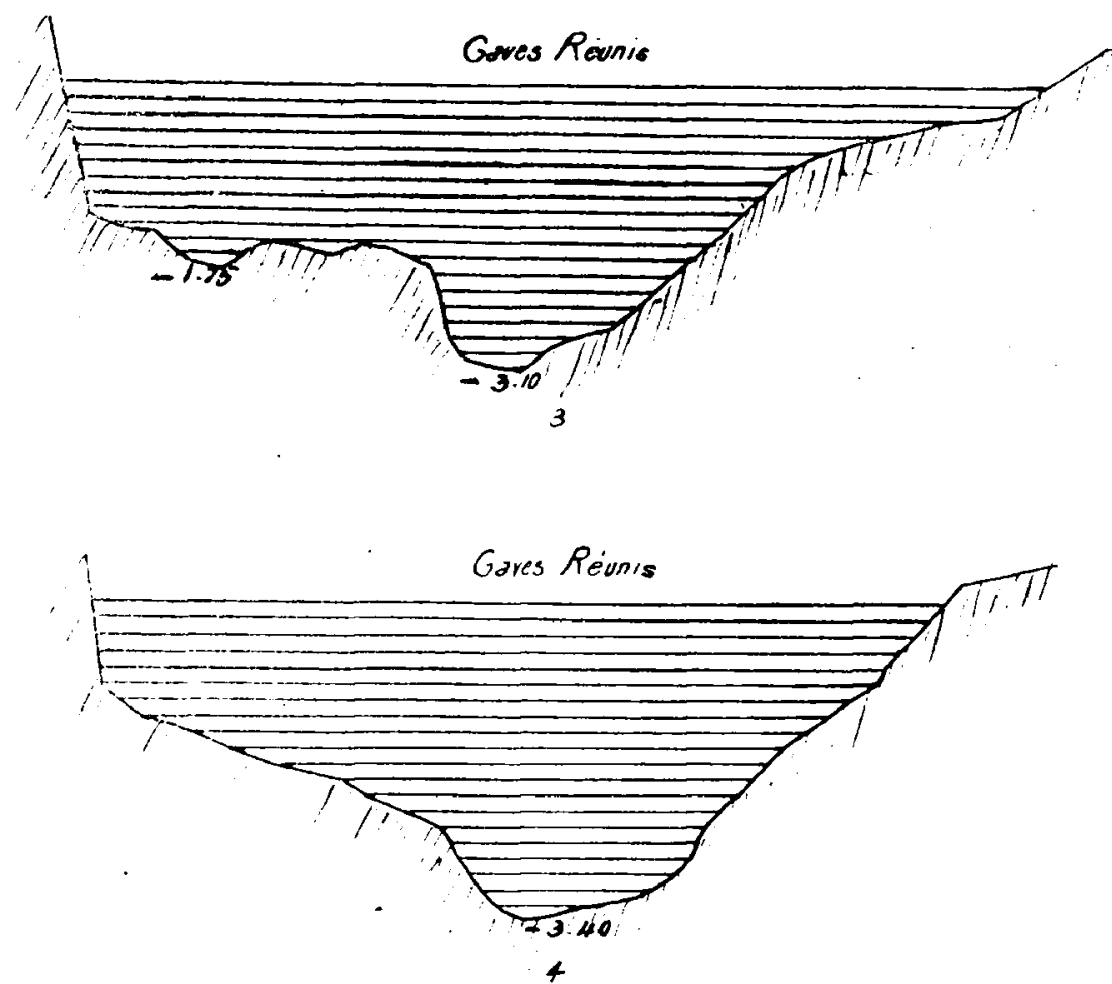

Fig. 3i. - Profils en Iravers des gaves de Pau et d'Oloron à leur point de jonction (1) et des Gaves réunis à 20,40 et 60 mètres en aval du confluent $(2),(3),(4)$.

(L'échelle des hauteurs est décuple de celle des largears). 
Il résulte de nos constatations que le gave d'Oloron, dans l'état actuel des choses, est sensiblement plus creux que le gave de Pau. Son sillon profond se peut suivre assez facilement par eaux claires, d'après un rapport du Brigadier des Eaux et Forêts d'Orthevielle (I). Il se continue dans les Gaves réunis sans qu'on puisse soupçonner une tendance du gave de Pau à prendre le fond de ce sillon comme niveau de base pour le modelage de son profil en long. Celui-ci se branche sur l'autre à angle aigu.

Ces faits ne laissent pas d'être inattendus, car, jusqu'ici, le gave d'Oloron était réputé l'affluent du gave de Pau. C'est ce qu'admet, en particulier, M. Jean Fischen, dans sa thèse récente du doctorat-ès-lettres (2). Il indique, d'ailleurs, comme pentes par kilomètre, dans la dernière partie du cours :

$$
\begin{aligned}
& \text { Gave de Pau...................... } \mathrm{o}^{\mathrm{m}} 74 \\
& \text { Gave d'Oloron................ } \quad \mathbf{I}^{\mathrm{m}} 48
\end{aligned}
$$

Il résulte, cependant, de relevés effectués par le Service des Forces hydrauliques que, pour les ro dernièrs kilomètres du profil en long de chacun des cours d'eau, il existerait une dénivellation de $8^{\mathrm{m}} 70$ pour le gave de Pau et de $9^{\mathrm{m}}$ 3o pour le gave d'Oloron. D'après ces données, la pente par kilomètre dans la section myriamétrique précédant le confluent serait :

$$
\begin{aligned}
& \text { Gave de Pau............... } \quad o^{m} 87 \\
& \text { Gave d'Oloron.............. } \mathrm{o}^{\mathbf{m}} 9^{3}
\end{aligned}
$$

Par conséquent, en $19^{29}$, il était admis que la déclivité était double pour le gave d'Oloron dont le profil en long s'inscrivait dans la concavité die celui du gave de Pau. Depuis, il est apparu que la différence des pentes en amont de la jonction serait de très faible importance et nous venons d'établir que le tributaire, au point de rencontre, était notablement plus creux que le cours d'eau réputé récepteur.

Il semble que, les choses étant ce que le font voir nos découvertes, le Saumon soit naturellement aiguillé, lors de son voyage de noces, vers le gave d'Oloron. Comme ce migrateur, du moins en hiver, nage en profondeur, la souille dont les caractéristiques ont été données exerce, selon toute vraisemblance, une influence directrice prépondérante.

Mais, quelles peuvent être les causes d'une situation qui, a priori, n'est pas naturelle?

Un examen altentif des lieux et de minuticuses recherches ont démontré, qu'effectivement, cette situation a été créée par l'Hommc. Les derniers sondages pratiqués ont mis en évidence l'existence d'un obstacle submergé, généralement ignoré, et dont l'origine paraît être ancienne.

A 2 ro mètres en aval du confluent des gaves de Pau et d'Oloron, à hauteur du moulin Laran, partant de la rive droite, trois rangées de forts

(1) Nous tenons à remercier ici le Brigadier des Eaux et Forêts Sancousse, qui, par son zèle et son intelligence, nous a permis de mener a bien la mission qui nous a été confiée.

(3) L'Adour et ses affluents. - Paris. Hachette, I929. 
piquets ont été plantées, formant un barrage de j mètres de largeur et 75 mètres de longueur obstruant le cours des Gaves réunis sur les trous quarts de sa largeur, mais laissant l'aulre quart libre du còté gauche, précisément à l'endroit où se trouve le sillon dont il a été parlé plus haut. Au pied de ce barrage est un blocage de grosses pierres (Fig. 36). Lne échancrure de 22 mètres a élé pratiquée en son milieu, il y a jo ans environ, pour y laisser passer les gabares chargées de sable.

L'urigine de cette installation est inconnue.

L'état des lieux avoisinants n'a révélé aucune prise d'eau, soit pour l'irrigation, soit pour l'alimentation d'un moulin. On ne peut non plus voir dans cet ouvrage, un travail de protection contre les inondations.

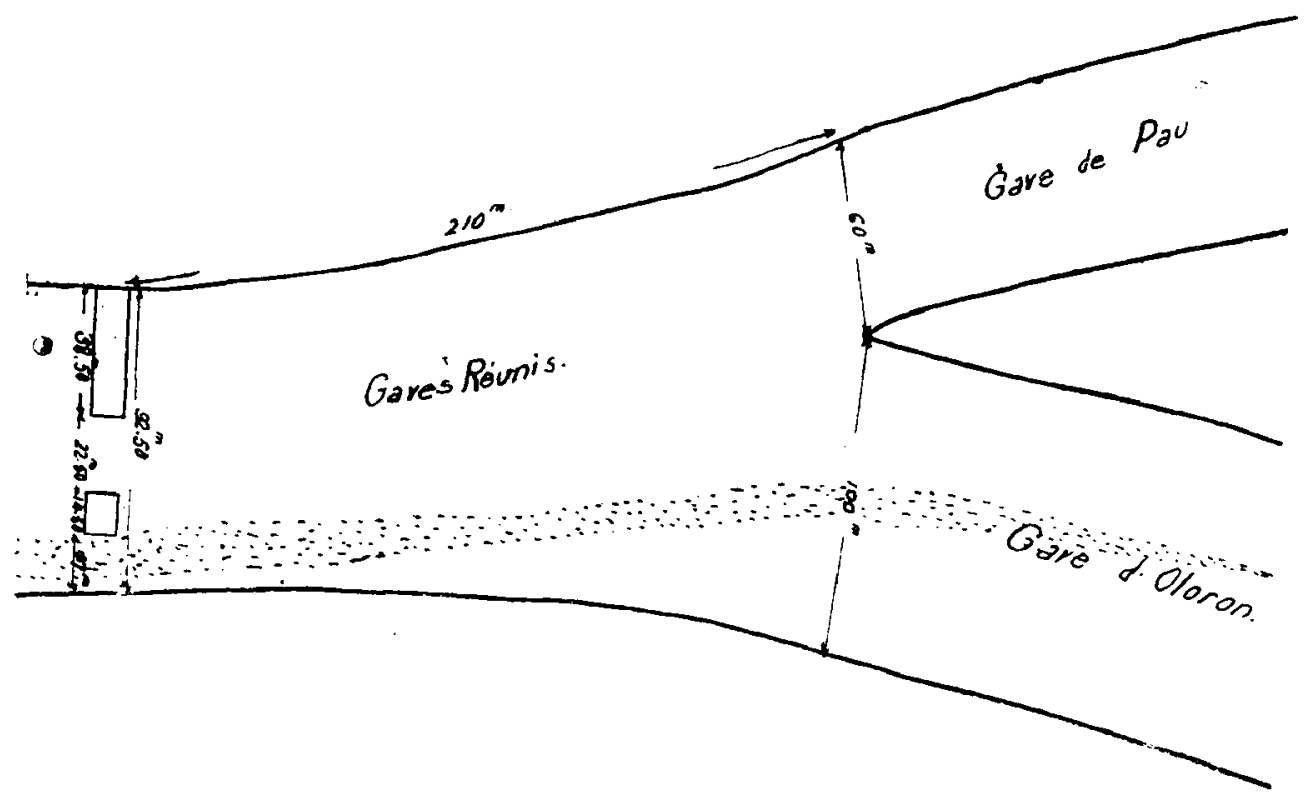

Fig. 36. - Confuent du gave de Pau et du ghave doloron. - Echelle : 1250 "

La partie surcreusée est figuré eu pointillés.

Il semble bien que cet aménagement ait été fait à fins halieutiques, dans le dessein d'entraver la remonte du Saumon dans le gave de Pau, au bénéfice du grave d’Oloron ( $\mathrm{I}$ ).

(1) Dans une requite adressée aux Etals le la l'rovince de Béarn, au sujet de la pêche

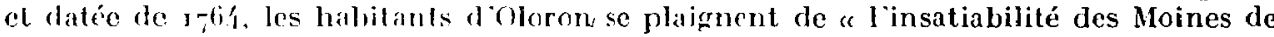
Sordes et des habitants do leyrchorade "qui, contrevenant aux ordonnances royales, leur ôtent ainsi la rescurce du goisson on birrant le Gave pour empêcher ceux-ci de monter.

A la suite d une vórification par le Maitre particulier, il est constaté qu'à r'4 de lieue cn remontant du port he l'eyrehorarde, il y a une "pessière " composce de gros piquets plautís dans le lit, soulenus par des piquets de pin mis en travers, etc...

Le (irand Viùtre de Guyenne, le sieur be: Bastarb, par ordonnance du 21 janvier 1765 , prescrit des mesures contre ces abus (Notes communiquées par M. le Conservateur des l.aux et Forits de Concr).

Tout porte i croire que k barrife submergé n'est autre que le soubassement de la

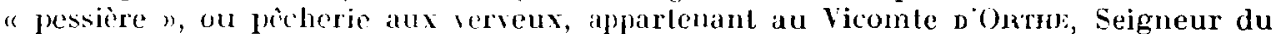
lieu et supprincée dans la scconcle paríe du xvin ${ }^{e}$ siècle. 
On conçoit que, depuis cette construction, le contingent de migrateurs ait été notablement plus élevé pour le second de ces cours d'eau.

Par ailleurs, notre trouvaille ne fournit-elle pas une explication salisfaisante pour l'anomalie du raccordement des deux gaves?

Nous nous trouvons - la forme même des profils en travers le montre, surtout avec l'exagération qu'implique le choix pour les hauteurs d'unc échelle décuple de celle des largeurs, - en présence d'un surcreusement de son lit par le gave d'Oloron, le gave de Pau étant resté inactif, en appa. rence au moins.

Bien que, d'après M. Fischer, la turbidité des deux cours d'eau soit réduite, même en temps de crue, on a des raisons de supposer un engorgement relativement récent de la section inférieure du gave de Pau, comme conséquence de l'existence du seuil constitué par le barrage submergé. L'hypothèse du banc de sable obstruant la section n'était donc pas fantaisiste. Le charriage étant faible, l'alluvionnement a dû être lent.

Quant à la souille, elle résulte aussi de l'interception partielle de la coulière naturelle. Dans la partie laissée libre, sur la côté gauche, le courant s'est trouvé renforcé. L'érosion a prélevé sur la section mouillée, en profondeur, ce que cetle dernière avait perdu en largeur quand fut créé lc seuil plus haut décrit. On ne voit pas qu'il y ait à chercher une autre explication des phénomènes observés.

La portion surcreuséc du gave d'Oloron n'est d'ailleurs, que la terminaison arrière du sillon de l'Adour et des Gaves réunis, autrement dit, sa queue. Le phénomène a commencé par l'aval.

Dans la masse des eaux dévalant, le rétrécissement artificiel du lit a déterminé la formation d'un flux à vitesse accélérée, issu du gave d'Oloron, laissant en quelque sorte à la traîne des courants marg̣inaux moins impétueux, provenant surtout du gave de Pau.

Sous l'action du flot le plus rapide, le lit ancien 's'est creusé ; le travail de déblaiement progressant, comme d'usage, de bas en haut.

Dans tous les cas, le régime différent des deux cours d'eau, tel qu'il se trouve présentement établi, n'a pu qu'accentuer l'attirance que le Saumon avait déjà pour le gave d'Oloron.

Les surfaces des bassins versants sont approximativement égales, savoir 2.5,6 kilomètres carrés pour le gave de Pau et 2.156 kilomètres carrés pour le gave d'Oloron. Mais les conditions d'alimentation et d'écoulement sont fort dissemblables, comme il ressort des indications fournies par les relevés établis, de I $_{222}$ à $\mathrm{r}_{9} 3 \mathrm{I}$, par le Service des Forces hydrauliques du Sud-Ouest, aux stations de jaugeage comparables $d u$ pont de Berenx, sur le gave de Pau, et d'Escos, sur celui d'Oloron.

Alors que le module du premier est de 7 I mètres cubes, celui du second atteint $9 \mathrm{I}$ mètres cubes.

Le débit mensuel moyen du gave d'Oloron l'emporte de 12 mètrescubes-seconde sur celui de l'autre. 
Enfin, pendant toute l'année, sauf en Juillet-Aout, le gave d'Oloron est mieux alimenté ; c'est seulement au fort de l'été que la fonte des névés et glaciers des Pyrénées entretient mieux le gave de Pau.

Tout concourt ainsi à faire du gave de Pau actuel l'affluent du gave d'Oloron. Cette inversion de la situation originelle, situation encore admise par les géographes, favorise évidemment le creusement de la souille dans les Gaves réunis et le décrochement des niveaux de base au point de jonction. Le cours d'eau récepteur s'est approfondi d'autant mieux qu'un obstacle artificiel a empêché le tributaire d'en faire autant.

Il est probable que, si on arrachait les piquets du barrage, si on détruisait le blocage existant, le raccordement normal ne tarderait pas à s'effectuer; la souille, très peu profonde, qui existe au point où l'ouvrage a été éventré sur 22 mètres il y a 50 ans environ, pour le passage des gabares, s'accentuerait et remonterait petit à petit le gave de Pau. On peut penser que l'on obtiendrait au bout d'un temps plus ou moins long, compte tenu des différents régimes, le raccordement tangentiel de son profil en long avec celui du gave d'Oloron.

Du point de vue piscicole, qui est celui nous préoccupant le plus, la réduction, puis l'arrêt de la remonte du Saumon dans le gave de Pau, semblent bien imputables, à la souille d'ahord, au barrage infranchissable de Castetarbe ensuite.

Avant la construction de ce dernier ouvrage on observait, en particulier, que le Saumon d'hiver n'avait pas l'habitude de s'engager dans le gave de Pau.

Ce fait s'explique par la rétention nivale, beaucoup plus sensible dans lo gave de Pau que dans celui d'Oloron (I). Le poisson amontant a toute raison de suivre, durant la saison froide, la coulière du second de ces cours d'eau qui est la plus creuse et la mieux alimentée.

On pêchait naguère à Orthez principalement des Saumons de printemps, parce qu'à cettc époque, la rétention nivale cessant, la différence d'alimentation entre les deux gaves étaił très atténuée et l'action exercée par la coulière réduite au minimum. Dès lors le Saumon de printemps, nageant entre deux eaux, avait plus de chances d'entrer dans le gave de Pau qui a, à ce moment-là, son débit maximum (2).

Mais, à partir de 1918 , date de la mise en service du barrage infranchissable de Castetarbe, la seule partie où le Saumon put aller s'est trouvée limitée au tronçon qui va du confluent au barrage, tronçon où le cours d'eau circule dạns une gorge très peu accessible, à parois rocheuses, abruptes. Les pêcheurs y allant très peu, ou mieux pas du tout, aucune

(1) Pour les quatre mois de Décembre, Janvier, Février, Mars, les débits moyens sont de 80 M. c. seconde pour le gave de Pau et de ino M. c. seconde pour le gave d'Oloron.

(2) Pour des mois de Mai et Juin le débit moven du gave de Pau est de ir6 M. c. seconde et celui du gave d'Oloron $122 \mathrm{M}$. c. seconde.

Ce sont les mois où les débits sont les plus forts et à peu près d'égale importance. 
capture n'y est faite et on a pu dire que le Saumon avait définitivement. déserté le gave de Pau.

De cet exposé, nous tirons donc les conclusions suivantes :

On ne saurait modifier le régime des gaves, mais il semble qu'on pourrait songer aujourd'hui à rétablir ou intensifier, dans celui de Pau, un courant de remonte par la suppression du barrage en piquets.

L'ouvrage de l'usine hydro-électrique de Castetarbe étant trop élevé pour être franchissable, la construction d'une échelle sur ce point est indispensable si on veut permettre aux Saumons de passer dans le bief supérieur et de gagner les frayères qu'il fréquentait autrefois entre Lourdes et Couarraze.

\title{
I,E MARCHÉ DE LA GARPE ${ }^{(1)}$
}

\author{
Par M. SORNAY \\ Conservateur des Faux et Forêts, \\ Chef de la Commission de Pisciculture des Bassins du Rhône et du Rhin.
}

La production des carpiculteurs français, comme il est normal, trouve son débouché principal en France, mais, néanmoins, une fraction non négligeable de cette production est exportée à l'étranger, principalement en Allemagne, pays qui demeure le plus gros consommateur européen de Carpes.

Or, quelle est la situation actuelle? Sur le marché intérieur, comme je l'ai exposé précédemment (2), la consommation du poisson d'eau douce se présente dans l'ensımble en régression continue, et la crise économique actuelle, par suite des restrictions qu'elle entraîne pour chacun, contribue ̀̀ accélérer cette réduction. Sur de rares points du territoire seulement on constate des faits d'ordre inverse. C'est ainsi qu'à Paris les arrivages de Carpes aux Halles centrales ont passé de 307.000 kilos en 1928, à 337.000 kilos en I $_{3} 3$ r. Notons avec satisfaction ce léger accroissement, malgré qu'il soit exceptionnel.

A l'étranger, la situation est plus difficile encore. En Allemagne, où se trouve le plus important marché d'Europe pour le poisson d'eau douce, les importations de Carpes ont sans doute atteint r.723.00o kilos en rg3o, alors qu'elles ne dépassaient pas r.000.000 kilos en 1927, mais il convient de remarquer que le chiffre de $\mathrm{rg} 3 \mathrm{I}$ se présente en baisse de $\mathrm{i} 5.000$ kilos sur celui de rg3o et que, pour les deux premiers trimestres de rg32, c'est une nouvelle réduction des importations atteignant I j0.000 kilos qu'il faut enregistrer pour la Carpe.

(1) Rapport présenté à la u Journée de la Carpe ", à Lyon, le 9 Novembre I93z.

(1) Voir Bulletin : - no 54 , Decembre $1932, p$. 180 . 